\title{
Numerical and analytical tests of quasi-integrability in modified sine-Gordon models
}

\author{
L.A. Ferreira ${ }^{a}$ and Wojtek J. Zakrzewski ${ }^{b}$ \\ ${ }^{a}$ Instituto de Física de São Carlos, IFSC/USP, Universidade de São Paulo, \\ Caixa Postal 369, CEP 13560-970, São Carlos-SP, Brazil \\ ${ }^{b}$ Department of Mathematical Sciences, University of Durham, \\ Durham DH1 3LE, U.K. \\ E-mail: laf@ifsc.usp.br, W.J.Zakrzewski@durham.ac.uk
}

ABSTRACT: Following our attempts to define quasi-integrability in which we related this concept to a particular symmetry of the two-soliton function we check this condition in three classes of modified sine-Gordon models in $(1+1)$ dimensions. We find that the numerical results seen in various scatterings of two solitons and in the time evolution of breather-like structures support our ideas about the symmetry of the field configurations and its effects on the anomalies of the conservation laws of the charges.

Keywords: Integrable Field Theories, Integrable Hierarchies, Integrable Equations in Physics, Solitons Monopoles and Instantons

ARXIV EPRINT: 1308.4412 


\section{Contents}

1 Introduction 1

2 The models $\quad 3$

2.1 First class of models 3

2.2 Second class of models 4

2.3 Third class of models 5

3 Quasi-integrability $\quad 6$

$\begin{array}{lll}3.1 & \text { Expansion around sine-Gordon } & 11\end{array}$

$\begin{array}{lll}3.1 .1 & \text { The two-soliton solutions } & 11\end{array}$

$\begin{array}{lll}3.1 .2 & \text { The breather solutions } & 13\end{array}$

$\begin{array}{ll}\text { 3.1.3 The parity versus dynamics argument for our models } & 14\end{array}$

4 Numerical simulations $\quad 16$

$\begin{array}{ll}4.1 \text { General considerations } & 17\end{array}$

$\begin{array}{ll}4.2 \text { Two soliton configurations } & 17\end{array}$

$\begin{array}{lll}4.3 & \text { Breather-like configurations } & 24\end{array}$

5 Further comments and some conclusions $\quad 28$

$\begin{array}{lr}\text { A The algebra } & 28\end{array}$

\section{Introduction}

In our recent work [1] and [2] we have tried to define the concept of quasi-integrability for field theories in $(1+1)$ dimensions. The idea to introduce such a concept is based on observation that many processes described by field theories which are not integrable possess properties which are very close to those seen in integrable theories. Thus, for example, in many processes involving scattering of (soliton-like) extended structures these structures do not alter significantly their shapes and the whole scattering process generates very little radiation. In fact, after developing the concept of quasi-integrability in $[1,2]$ we became aware of previous observations of elastic scattering of solitons in some nonintegrable theories [3, 4], which reinforces the relevance of the development of such concepts.

In our recent work we have looked in detail, both numerically and analytically, at the scattering of solitons in two classes of models in $(1+1)$ dimensions, namely the generalized models of the sine-Gordon type as introduced by Bazeia et al. [5] and the generalized models of the Nonlinear Schrödinger type (in which the usual NLS potential $|\psi|^{4}$ is generalized to $\left.|\psi|^{4+\varepsilon}\right)$. Both models depend on a new parameter $(\varepsilon)$ but when this parameter vanishes they become the familiar integrable models (the sine-Gordon and NLS respectively). 
Our numerical results have shown that, in contradistinction to the integrable cases, the integrability-like constraints hold only for some field configurations and that they hold for the field configurations involving the scatterings of two solitons. Hence we have tried to formulate the concept by expanding the models around their integrable points and specializing the discussion by looking at field configurations involving two solitons. Our results have then shown that some field configurations are very special; namely, when the field configurations possessed an extra parity-like symmetry the models, for these configurations, possessed extra conserved quantities (very much like their integrable cousins). For other configurations these quantities were not conserved but were very small - with the meaning of 'small' very dependent on the field configuration and on the closeness of the model to being integrable. Hence our views on quasi-integrability were very much tied up with these extra symmetries and this closeness.

To investigate this further we have decided to look at other topological models, in $(1+1)$ dimensions; to check whether this relation of the quasi-integrability to the symmetry of the field configuration holds also in other models.

Hence we have decided to look at this in three topological models, dependent on a free parameter $\varepsilon$, which for a specific value of this parameter (namely $\varepsilon=0$ ) reduce to the sine-Gordon model. The models were constructed using the procedure discussed in [6], which, in fact, is a generalization of the procedure discussed in [5]. The first two models are obtained by two different changes of variables from the field of the sine-Gordon model. In each case the one soliton field configuration connects the vacua at 0 and $\frac{\pi}{2}$. In one case there is a further vacuum at $-\frac{\pi}{2}$ irrespective of the value of the free parameter $\varepsilon$ while in the other one all other vacua depend on $\varepsilon$. Hence the first model allows for the symmetry of the two-soliton field configuration while the second one does not. Finally, we have considered also a third model, which depends on two parameters. When one of these two parameters is zero we have a situation like in the first class of models but when both parameters are nonzero we can study the transition from one regime to the other one and its effect on the properties of the anomaly.

The solitons reflect in their scatterings and so their effects on the anomalies are restricted only to the period when they are close together. However, we can also look at the configurations resembling breathers of the sine-Gordon model. Such configurations involve one soliton and one anti-soliton which are bound together (their energy is less than the energy of a soliton and an anti-soliton). Hence they are always in interaction and during this interaction they radiate some energy. Thus the time evolution of their anomalies is stronger and so they can tell us more of the relevance of our symmetry on quasi-integrability. Hence in the sequel we look at such configurations in the third class of models.

The paper is organized as follows. Section 2 presents our models, their properties and discusses their two soliton field configurations. Section 3 recalls our ideas on quasiintegrability and their relation to the symmetry of the two-soliton configuration. Our ideas are then tested, as described in section 4, in numerical simulations of the scattering properties of two soliton configurations and time evolution of breather-like configurations. We show that they support our expectations that the anomaly effects are smaller in models with symmetry and more pronounced in the models in which this symmetry is broken. We finish the paper with further comments and some conclusions, given in section 5 . 


\section{The models}

The three classes of models we consider in this paper are based on the Lagrangian density given by

$$
L=\frac{1}{2}\left(\left(\partial_{t} \phi\right)^{2}-\left(\partial_{x} \phi\right)^{2}\right)-V(\phi)
$$

where the potentials $V(\phi)$ are conveniently chosen by the procedure discussed in [6], which we now briefly explain. Following that procedure we take the field $\psi$ of the sine-Gordon model, defined by the Lagrangian

$$
L_{\mathrm{SG}}=\frac{1}{2}\left(\left(\partial_{t} \psi\right)^{2}-\left(\partial_{x} \psi\right)^{2}\right)-V_{\mathrm{SG}} ; \quad V_{\mathrm{SG}}=\frac{1}{8} \sin ^{2}(2 \psi)
$$

The static one-soliton solutions of the sine-Gordon model, given by

$$
\psi=\operatorname{ArcTan}\left(e^{ \pm\left(x-x_{0}\right)}\right)
$$

are solutions of the so-called BPS equation

$$
\partial_{x} \psi= \pm \sqrt{2 V_{\mathrm{SG}}}
$$

Indeed, any solution of (2.4) is a solution of the static Euler-Lagrange equation associated to $(2.2)$.

Suppose now we introduce a field $\phi$ as a given transformation of the sine-Gordon field $\psi$, i.e. $\phi \equiv \phi(\psi)$. Then, it follows from (2.4) that

$$
\partial_{x} \phi= \pm \sqrt{2 V(\phi)}
$$

with

$$
V(\phi) \equiv\left(\frac{d \phi}{d \psi}\right)^{2} V_{\mathrm{SG}}=\frac{1}{8} \sin ^{2}[2 \psi(\phi)]\left(\frac{d \phi}{d \psi}\right)^{2}
$$

If one takes the theory (2.1) with potential given by (2.6), then it follows that static solutions of (2.5) are solutions of the static Euler-Lagrange equation associated to (2.1). Therefore, the mapping $\phi \equiv \phi(\psi)$, maps BPS solutions of the sine-Gordon model into BPS solutions of the theory $(2.1)$.

In this paper we shall consider three theories of the type (2.1), with potentials given by (2.6), for three particular choices of mappings $\phi \equiv \phi(\psi)$, which we now describe.

\subsection{First class of models}

In the first class of models we perform the change of variables:

$$
\phi(\psi)=\frac{\psi}{1+\varepsilon\left(\frac{\pi^{2}}{4}-\psi^{2}\right)} .
$$

where $\varepsilon$ is a free parameter which has to satisfy $\varepsilon>-\frac{4}{\pi^{2}}$ and when $\varepsilon=0$ we have the identity map, and so (2.1) becomes the sine-Gordon model. Note that $\phi(\psi=0)=0$ and $\phi\left(\psi= \pm \frac{\pi}{2}\right)= \pm \frac{\pi}{2}$. Therefore, the three neighboring vacua of the sine-Gordon model, 


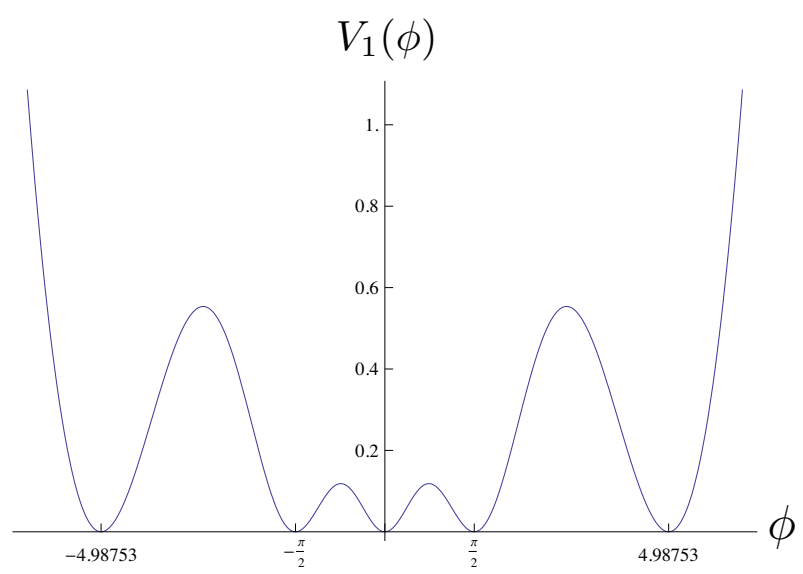

Figure 1. Plot of the potential $V_{1}(\phi)$, given in (2.10), against $\phi$ for $\varepsilon=0.05$. Note that $V_{1}(\phi)$ is invariant under the change $\phi \rightarrow-\phi$, but its infinite number of vacua are not equally spaced. Therefore, a one-soliton solution tunneling between the vacua $\phi=0$ and $\phi=\frac{\pi}{2}$ have a mass which is different from, for instance, that of the one-soliton tunneling between the vacua $\phi=\frac{\pi}{2}$ and $\phi=4.98753$.

namely, $\psi=0, \pm \frac{\pi}{2}$, will also be vacua of the new model. The same is not true however for the other SG vacua. The inverse of this change of variables is provided by

$$
\psi=\frac{2 a \phi}{1+\Gamma}, \quad \text { with } \quad a=1+\frac{\varepsilon \pi^{2}}{4} \quad \Gamma=\sqrt{1+4 \varepsilon a \phi^{2}}
$$

One can easily check that

$$
\frac{d \psi}{d \phi}=\frac{2 a}{\Gamma(1+\Gamma)}
$$

Therefore, our first class of theory is defined by the Lagrangian (2.1) with potential given by

$$
V_{1}(\phi)=\frac{1}{32 a^{2}} \Gamma^{2}(1+\Gamma)^{2} \sin ^{2}\left(\frac{4 a \phi}{1+\Gamma}\right)
$$

Note that static one soliton solution of such a theory is given by (2.7) in which $\psi$ is given by (2.3). We provide in figure 1 a plot of the potential (2.10) for $\varepsilon=0.05$.

\subsection{Second class of models}

In this case we make a change of variables:

$$
\phi=\frac{b \psi}{1+\varepsilon \psi} ; \quad \psi=\frac{\phi}{(b-\varepsilon \phi)} ; \quad \text { with } \quad b=1+\varepsilon \frac{\pi}{2}
$$

where the choice of the parameter $b$ such that $\phi(\psi=0)=0$ and $\phi\left(\psi=\frac{\pi}{2}\right)=\frac{\pi}{2}$. Therefore, one has

$$
\frac{d \phi}{d \psi}=\frac{(b-\varepsilon \phi)^{2}}{b}
$$

and so our second class of theories is defined by the Lagrangian (2.1) with the potential being

$$
V_{2}(\phi)=\frac{1}{8} \frac{(b-\varepsilon \phi)^{4}}{b^{2}} \sin ^{2}\left(\frac{2 \phi}{b-\varepsilon \phi}\right)
$$




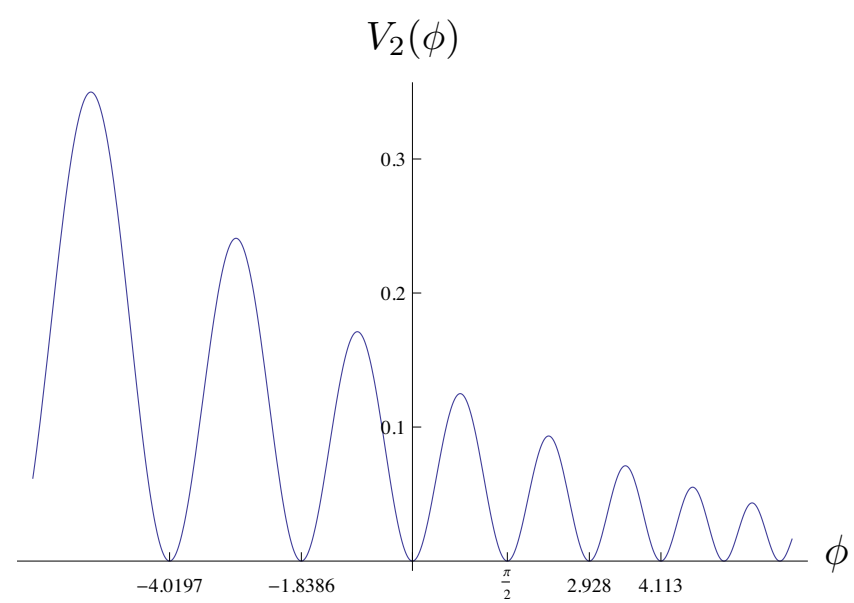

Figure 2. Plot of the potential $V_{2}(\phi)$, given in (2.13), against $\phi$ for $\varepsilon=0.05$. Contrary to $V_{1}$ this potential $V_{2}$ is not invariant under the change $\phi \rightarrow-\phi$, and its (infinite number) vacua are not equally spaced. Therefore, the one-soliton solutions tunneling between neighbouring vacua do not have the same mass.

Again the static one soliton solution of this model is given by (2.11) in which $\psi$ is given by (2.3). We give in figure 2 a plot of the potential (2.13) for $\varepsilon=0.05$.

\subsection{Third class of models}

Given the very different behaviour of both models presented above, and given the numerical results which we obtained for them which will be discussed in section 4 , we have decided to look also at a model which interpolates between the two of them. To study this in more detail we have decided to construct a third model (with two parameters). This would allows us to study the transition from one behaviour to the other as we change the parameters. We then introduce the third class of models based on the change of variables

$$
\psi(\phi)=\frac{c \phi}{\sqrt{1+\varepsilon \phi(\phi-2 \gamma)}} .
$$

where

$$
c=\sqrt{1+\varepsilon \pi\left(\frac{\pi}{4}-\gamma\right)},
$$

The parameter $c$ was chosen such that $\phi(\psi=0)=0$ and $\phi\left(\psi=\frac{\pi}{2}\right)=\frac{\pi}{2}$. The free parameters are $\varepsilon$ and $\gamma$.

It then follows that the third class of theories is defined by the Lagrangian (2.1) with the potential being given by

$$
V_{3}(\phi)=\frac{1}{8} \frac{(1+\varepsilon \phi(\phi-2 \gamma))^{3}}{c^{2}(1-\varepsilon \gamma \phi)^{2}} \sin ^{2}(2 \psi(\phi)),
$$

where $\psi(\phi)$ is given by (2.14). Note that when $\gamma=0$ the model possesses the symmetry $\phi \rightarrow-\phi$, which will be desirable for quasi-integrability as we discuss it further in the paper. 

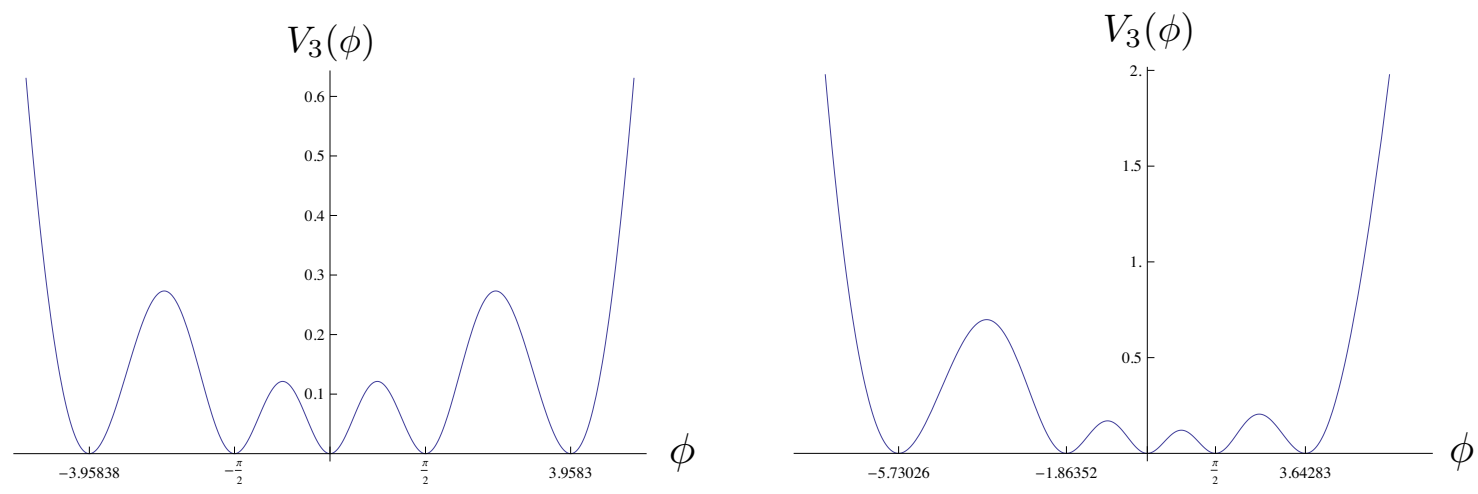

Figure 3. Plots of the potential $V_{3}(\phi)$, given in (2.16), against $\phi$ for $\varepsilon=0.05$, with $\gamma=0$ for the left plot, and $\gamma=1$ for the right plot. Note that this class of potentials interpolates between the first class (for $\gamma=0$ ) and the second class (for $\gamma \neq 0$ ). Note that, for $\gamma=0, V_{3}$ is symmetric under the change $\phi \rightarrow-\phi$, but not for other values of $\gamma$, and as long as $\varepsilon \neq 0$, the infinite number of vacua are not equally spaced, leading to one-soliton solutions of different masses.

Then the $\gamma=0$ case resembles a little the first class models given by the potential (2.10). The case where $\gamma \neq 0$ we do not have any symmetry and so the model is more like the models of the second class, given by the potential (2.13). When $\varepsilon=0$, the parameter $\gamma$ becomes irrelevant and we have the sine-Gordon model. Consequently, this third class of models is a good place to study the transition mentioned above.

\section{Quasi-integrability}

Next we perform an analytical study of the properties of our models using the techniques usually employed in integral field theories to construct quantities which are quasi-conserved as will be explained below. We follow the ideas put forward in our previous works on quasiintegrability $[1,2]$.

We consider a scalar field theory for a real scalar field $\phi$ in a generic potential $V(\phi)$. As in [1] we introduce the Lax potentials, based on a $s l(2)$ loop algebra:

$$
\begin{aligned}
& A_{+}=\frac{1}{2}\left[\left(\omega^{2} V-m\right) b_{1}-i \omega \frac{d V}{d \phi} F_{1}\right], \\
& A_{-}=\frac{1}{2} b_{-1}-\frac{i}{2} \omega \partial_{-} \phi F_{0},
\end{aligned}
$$

where we have used light-cone variables

$$
x_{ \pm}=\frac{1}{2}(t \pm x) \quad \text { with } \quad \partial_{ \pm}=\partial_{t} \pm \partial_{x} \quad \text { and } \quad \partial_{+} \partial_{-}=\partial_{t}^{2}-\partial_{x}^{2} \equiv \partial^{2} .
$$

The $s l(2)$ loop algebra generators $b_{n}$ and $F_{n}$ are defined in the appendix $\mathrm{A}$, and $\omega$ and $m$ are free parameters which do not appear in the Lagrangian or the equations of motion of the theory. One can easily check that the curvature of the connection (3.1) is given by

$$
F_{+-} \equiv \partial_{+} A_{-}-\partial_{-} A_{+}+\left[A_{+}, A_{-}\right]=X F_{1}-\frac{i \omega}{2}\left[\partial^{2} \phi+\frac{\partial V}{\partial \phi}\right] F_{0}
$$


with

$$
X=\frac{i \omega}{2} \partial_{-} \phi\left[\frac{d^{2} V}{d \phi^{2}}+\omega^{2} V-m\right] .
$$

Note that when the equations of motion, following from (2.1), are satisfied, i.e.

$$
\partial^{2} \phi+\frac{\partial V}{\partial \phi}=0
$$

the term proportional to the Lie algebra generator $F_{0}$ vanishes. In addition, the quantity $X$ vanishes for the sine-Gordon potential

$$
V_{\mathrm{SG}}=\frac{1}{16}[1-\cos (4 \phi)]
$$

if one chooses the parameters $m$ and $\omega$ as $m=1$ and $\omega=4 .^{1}$ Therefore, the curvature vanishes for the sine-Gordon theory, and that is what makes this theory an integrable field theory in $(1+1)$ dimensions. For any other choice of the potential $V(\phi)$, however, the curvature does not vanish. Following the steps given in [1] now analyze what can be said about conservation laws in a theory with non-vanishing $X$, or equivalently $F_{+-}$.

We start by recalling the fact that the generator $b_{-1}$ is a semi-simple element in the sense that its adjoint action splits the $s l(2)$ loop algebra $\mathcal{G}$ into its kernel and image as follows:

$$
\mathcal{G}=\text { Ker }+\operatorname{Im}, \quad\left[b_{-1}, \text { Ker }\right]=0, \quad \operatorname{Im}=\left[b_{-1}, \mathcal{G}\right]
$$

with the important property that Ker and Im have no common elements. An important ingredient of the method is that our $\operatorname{sl}(2)$ loop algebra $\mathcal{G}$ is graded by the grading operator $d=T_{3}+2 \lambda \frac{d}{d \lambda}$ (see appendix A for details), with the integer grades $n$ being determined as

$$
\mathcal{G}=\sum_{n} \mathcal{G}_{n} ; \quad\left[\mathcal{G}_{m}, \mathcal{G}_{n}\right] \subset \mathcal{G}_{m+n} ; \quad\left[d, \mathcal{G}_{n}\right]=n \mathcal{G}_{n}
$$

Next we perform a gauge transformation

$$
A_{\mu} \rightarrow a_{\mu}=g A_{\mu} g^{-1}-\partial_{\mu} g g^{-1}
$$

with the group element $g$ being an exponentiation of generators lying in the positive grade subspace generated by the $F_{n}$ 's, i.e.,

$$
g=\exp \left[\sum_{n=1}^{\infty} \mathcal{F}^{(n)}\right] \quad \text { with } \quad \mathcal{F}^{(n)}=\zeta_{n} F_{n}
$$

and $\zeta_{n}$, being the parameters of the transformation, which will be determined as we will explain below. Under (3.9) the curvature (3.3) transforms as

$$
F_{+-} \rightarrow g F_{+-} g^{-1}=\partial_{+} a_{-}-\partial_{-} a_{+}+\left[a_{+}, a_{-}\right]=X g F_{1} g^{-1},
$$

where we have used the equations of motion (3.5) to drop the term proportional to $F_{0}$ in (3.3).

\footnotetext{
${ }^{1}$ These values change if one modifies the sine-Gordon coupling constant and the zero of the potential.
} 
The component $A_{-}$of the connection (3.1) has terms with grades 0 and -1 . Therefore, under (3.9) it gets transformed into $a_{-}$which has terms with grades ranging from -1 to $+\infty$. Decomposing $a_{-}$into grades we get from (3.9) and (3.10) that

$$
\begin{aligned}
a_{-}= & \frac{1}{2} b_{-1} \\
& -\frac{1}{2}\left[b_{-1}, \mathcal{F}^{(1)}\right]-\frac{i}{2} \omega \partial_{-} \phi F_{0} \\
& -\frac{1}{2}\left[b_{-1}, \mathcal{F}^{(2)}\right]+\frac{1}{4}\left[\left[b_{-1}, \mathcal{F}^{(1)}\right], \mathcal{F}^{(1)}\right]-\frac{i}{2} \omega \partial_{-} \phi\left[\mathcal{F}^{(1)}, F_{0}\right]-\partial_{-} \mathcal{F}^{(1)} \\
& \vdots \\
& -\frac{1}{2}\left[b_{-1}, \mathcal{F}^{(n)}\right]+\ldots
\end{aligned}
$$

Next we note that we can make the component of $a_{-}$in the direction of $F_{n-1}$ vanish by choosing the parameters $\zeta_{n}$. In fact we can do this recursively. In particular, $\zeta_{1}=\frac{i}{2} \omega \partial_{-} \phi$, and so on. Thus our gauge transformation (3.10) has rotated the component $a_{-}$into the abelian subalgebra generated by the $b_{2 n+1}$ 's. Note that in this procedure we have not used the equations of motion (3.5). Thus

$$
a_{-}=\frac{1}{2} b_{-1}+\sum_{n=0}^{\infty} a_{-}^{(2 n+1)} b_{2 n+1}
$$

Next we note that, even when the equations of motion (3.5) are used, the component $a_{+}$of the connection does not get rotated into the abelian subalgebra generated by the $b_{2 n+1}$ 's (i.e. the kernel), as it has components in the image too, i.e. it is of the form:

$$
a_{+}=\sum_{n=0}^{\infty} a_{+}^{(2 n+1)} b_{2 n+1}+\sum_{n=2}^{\infty} c_{+}^{(n)} F_{n} .
$$

It turns out that the coefficients $c_{+}^{(n)}$ are all proportional to $X$, given in (3.4), and so they vanish for the sine-Gordon theory. As we shall see below this vanishing of $X$ is what is responsible for the integrability of the sine-Gordon theory as it possesses an infinite set of exact conservation laws.

The next step of our procedure, as discussed in [1] involves the decomposition of the curvature (3.11) into two components, one lying in the abelian subalgebra generated by $b_{2 n+1}$ 's and the other lying in the subspace generated by $F_{n}$ 's. Thus,

$$
g F_{1} g^{-1}=\sum_{n=0}^{\infty} \gamma^{(2 n+1)} b_{2 n+1}+\text { terms proportional to } F_{n} \text { 's }
$$

Since $a_{-}$lies in the kernel it follows that the commutator $\left[a_{+}, a_{-}\right]$does not produce terms in the kernel. Therefore, the terms in the direction of the $b_{2 n+1}$ 's of the transformed curvature (3.11) lead to the equations of the form

$$
\partial_{+} a_{-}^{(2 n+1)}-\partial_{-} a_{+}^{(2 n+1)}=X \gamma^{(2 n+1)} \quad n=0,1,2, \ldots
$$

with the anomaly $X$ given in (3.4). 
If we rewrite this using $x$ and $t$ variables we find that (3.16) takes the form $\partial_{t} a_{x}^{(2 n+1)}-$ $\partial_{x} a_{t}^{(2 n+1)}=-\frac{1}{2} X \gamma^{(2 n+1)}$, and so we find that (see (3.2))

$$
\frac{d Q^{(2 n+1)}}{d t}=-\frac{1}{2} \alpha^{(2 n+1)}+\left.a_{t}^{(2 n+1)}\right|_{x=-\infty} ^{x=\infty},
$$

where

$$
Q^{(2 n+1)} \equiv \int_{-\infty}^{\infty} d x a_{x}^{(2 n+1)}, \quad \quad \alpha^{(2 n+1)} \equiv \int_{-\infty}^{\infty} d x X \gamma^{(2 n+1)} .
$$

For finite energy solutions the fields $\phi$ have to go to a vacuum configuration at spatial infinity and, in general, this condition is enough for the values of $a_{t}^{(2 n+1)}$ at $x= \pm \infty$ to be equal. Therefore, the non-conservation of the charges is due to the non-vanishing of the anomalies $\alpha^{(2 n+1)}$, and so of the non-vanishing of the quantity $X$ given in (3.4).

To proceed further we introduce the following order two automorphism of the $\operatorname{sl}(2)$ loop algebra

$$
\Sigma\left(b_{2 n+1}\right)=-b_{2 n+1}, \quad \Sigma\left(F_{2 n}\right)=-F_{2 n}, \quad \Sigma\left(F_{2 n+1}\right)=F_{2 n+1}
$$

which is, in fact, the same as (see the appendix A)

$$
\Sigma\left(T_{3}\right)=-T_{3}, \quad \Sigma\left(T_{ \pm}\right)=-\lambda^{ \pm 1} T_{\mp}
$$

Note that (3.20) is an order two automorphism of the finite $\operatorname{sl}(2)$ algebra (A.1), with $\lambda$ being any constant and not necessarily the spectral parameter of the $\operatorname{sl}(2)$ loop algebra (A.2)(A.3). At the same time we introduce the space-time parity transformation

$$
P: \quad(\tilde{x}, \tilde{t}) \rightarrow(-\tilde{x},-\tilde{t}) \quad \text { with } \quad \tilde{x}=x-x_{\Delta} \quad \tilde{t}=t-t_{\Delta},
$$

where $x_{\Delta}$ and $t_{\Delta}$ are constants which, as we will see later, are determined by the particular solution under consideration. Even though the values of these constants vary from a solution to a solution, the argument we are presenting is independent of their values.

Next we make an important hypothesis that will have to be verified for each particular solution of the equations of motion that we want to study. We assume that, under the parity $P$, the scalar field $\phi$, evaluated on the solution under consideration, transforms as

$$
\phi \rightarrow-\phi+\text { const. }
$$

Thus, from (3.1), we see that

$$
\Sigma\left(A_{-}\right)=-A_{-} \quad P\left(A_{-}\right)=A_{-}
$$

and so

$$
\Omega\left(A_{-}\right)=-A_{-} \quad \text { with } \quad \Omega \equiv \Sigma P .
$$

Next we take the even part of (3.12) under $\Omega$ and split it in its grade components. From the first line of (3.12) we find that

$$
\left.(1+\Omega) a_{-}\right|_{-1}=0 .
$$


The second line of (3.12) then implies that

$$
\left.(1+\Omega) a_{-}\right|_{0}=-\frac{1}{2}\left[b_{-1},(1-\Omega) \mathcal{F}^{(1)}\right] .
$$

Note that, since we have rotated $a_{-}$into the Ker, the l.h.s. of (3.26) has to lie in this Ker. However, the r.h.s. of (3.26) lies in the Im. Therefore both sides have to vanish, i.e.

$$
\left.(1+\Omega) a_{-}\right|_{0}=0, \quad(1-\Omega) \mathcal{F}^{(1)}=0 .
$$

Note also, using the fact that $\mathcal{F}^{(1)}$ is even under $\Omega$, that the third line of (3.12) gives

$$
\left.(1+\Omega) a_{-}\right|_{1}=-\frac{1}{2}\left[b_{-1},(1-\Omega) \mathcal{F}^{(2)}\right]
$$

and so using the same arguments as above we see that

$$
\left.(1+\Omega) a_{-}\right|_{1}=0, \quad(1-\Omega) \mathcal{F}^{(2)}=0 .
$$

These arguments can now be repeated and so we see that all $\mathcal{F}^{(n)}$ are even under $\Omega$ and so, in consequence, the group element performing the gauge transformation (3.9) satisfies

$$
\Omega(g)=g .
$$

The Killing form of the $s l(2)$ loop algebra is given by

$$
\operatorname{Tr}\left(b_{2 m+1} b_{2 n+1}\right)=c \delta_{2(m+n)+2,0}, \quad \operatorname{Tr}\left(b_{2 m+1} F_{n}\right)=0
$$

for some constant $c$. Thus, using (3.31), we find from (3.15) that

$$
\gamma^{(2 n+1)}=\frac{1}{c} \operatorname{Tr}\left(g F_{1} g^{-1} b_{-2 n-1}\right)=-\frac{1}{c} \operatorname{Tr}\left(\Sigma(g) F_{1} \Sigma\left(g^{-1}\right) b_{-2 n-1}\right)
$$

and so

$$
P\left(\gamma^{(2 n+1)}\right)=-\frac{1}{c} \operatorname{Tr}\left(\Omega(g) F_{1} \Omega\left(g^{-1}\right) b_{-2 n-1}\right)=-\frac{1}{c} \operatorname{Tr}\left(g F_{1} g^{-1} b_{-2 n-1}\right)=-\gamma^{(2 n+1)} .
$$

Next we make our second hypothesis that will have to be verified for each particular potential; namely, that the quantity $X$ defined in (3.4) satisfies

$$
P(X)=X
$$

This can be achieved, for example, if the potential $V$, evaluated on the solution, is even under the parity $P$ given in (3.21). If this is true it then follows that

$$
\int_{-\tilde{t}_{0}}^{\tilde{t}_{0}} d t \int_{-\tilde{x}_{0}}^{\tilde{x}_{0}} d x X \gamma^{(2 n+1)}=0,
$$

where $\tilde{t}_{0}$ and $\tilde{x}_{0}$ are any given fixed values of the shifted time $\tilde{t}$ and space coordinate $\tilde{x}$, respectively, introduced in (3.21). If this is the case then, by taking $\tilde{x}_{0} \rightarrow \infty$, we conclude 
that the non-conserved charges (3.18) satisfy the following mirror time-symmetry around the point: $t_{\Delta}$.

$$
Q^{(2 n+1)}\left(t=\tilde{t}_{0}+t_{\Delta}\right)=Q^{(2 n+1)}\left(t=-\tilde{t}_{0}+t_{\Delta}\right) \quad n=0,1,2, \ldots
$$

Another infinite set of quasi-conserved charges, satisfying (3.36), can be constructed by a connection like that given in (3.1) but with $x_{+}$and $x_{-}$exchanging roles, and the grades changing sign. Then one performs a gauge transformation like in (3.9) but with the group element $g$ being an exponentiation like in (3.10) but involving negative grade generators $F_{-n}$. See [1] for the details of that construction.

Summarizing, we conclude that

1. If we have a two-soliton-like or a breather-like solution of the equations of motion (3.5), transforming under the space-time parity (3.21) as in (3.22), i.e.

$$
P(\phi)=-\phi+\text { const. }
$$

2. And if the potential, evaluated on such a solution is even under the parity, i.e.

$$
P(V)=V
$$

such that (3.34) holds true,

3. Then we have an infinite set of quantities which are conserved asymptotically, i.e.

$$
Q^{(2 n+1)}(t=+\infty)=Q^{(2 n+1)}(t=-\infty)
$$

So, the values of these charges in the infinite past, before the scattering of the solitons, are the same as in the infinite future, after the scattering. For the breather solutions such charges are symmetric by reflection around $t=t_{\Delta}$ (see (3.36)), and in fact oscillate in time. Theories possessing such properties we call quasi-integrable theories.

\subsection{Expansion around sine-Gordon}

Here we analyze under what circumstances one can satisfy the conditions given above and so have quasi-integrability. We start by looking at the properties of the two-soliton and breather solutions of the sine-Gordon model. For the potential (3.6) the equation of motion (3.5) becomes the sine-Gordon equation

$$
\partial^{2} \phi_{0}+\frac{1}{4} \sin \left(4 \phi_{0}\right)=0
$$

\subsubsection{The two-soliton solutions}

The two-soliton solution of (3.40) is given by

$$
\phi_{0}=\operatorname{ArcTan} \frac{\operatorname{Im} \tau_{0}}{\operatorname{Re} \tau_{0}},
$$


where the $\tau_{0}$ function is given by

$$
\tau_{0}=1+i e^{\Gamma_{1}}+i e^{\Gamma_{2}}-\gamma e^{\Gamma_{1}+\Gamma_{2}}
$$

with

$$
\Gamma_{i}=\eta_{i} \frac{\left(x-v_{i} t-x_{0}^{(i)}\right)}{\sqrt{1-v_{i}^{2}}} \quad i=1,2
$$

and

$$
\gamma=\left(\tanh \left(\frac{\alpha_{2}-\alpha_{1}}{2}\right)\right)^{2 \varepsilon_{1} \varepsilon_{2}}
$$

and where $\eta_{i}= \pm 1$, for kink and anti-kink. Here, $v_{i}=\tanh \alpha_{i}$, is the velocity (in units of the speed of light) of the kink (anti-kink) $i$.

Note that we can write the solution (3.41) as

$$
\phi_{0}=\operatorname{ArcTan}\left(-\frac{1}{\sigma}\right)
$$

where

$$
\sigma \equiv \sqrt{\gamma} \frac{\sinh z_{+}}{\cosh z_{-}}
$$

and

$$
z_{+}=\frac{\Gamma_{1}+\Gamma_{2}}{2}+\delta, \quad z_{-}=\frac{\Gamma_{1}-\Gamma_{2}}{2}, \quad \delta=\ln \sqrt{\gamma}
$$

Note also that as $z_{+}$and $z_{-}$are linear functions of $x$ and $t$ they can be taken as new coordinates of the space-time. They are, of course, independent of each other due to the fact that in a two-soliton solution we can never put $v_{1}=v_{2}$. Indeed, if that happens the two-soliton solution reduces to a one-soliton solution for the case $\eta_{1} \eta_{2}=1$, and one gets a divergent factor $(\gamma \rightarrow \infty)$, for $\eta_{1} \eta_{2}=-1$. In addition, we see from (3.41) that

$$
\sin \phi_{0}=\frac{\operatorname{Im} \tau_{0}}{\left|\tau_{0}\right|}=\frac{1}{\sqrt{1+\sigma^{2}}}, \quad \cos \phi_{0}=\frac{\operatorname{Re} \tau_{0}}{\left|\tau_{0}\right|}=-\frac{\sigma}{\sqrt{1+\sigma^{2}}}
$$

Thus, under the space-time parity transformation

$$
P: \quad\left(z_{+}, z_{-}\right) \rightarrow\left(-z_{+},-z_{-}\right)
$$

we see that $P(\sigma)=-\sigma$, and so, taking the domain of ArcTan to be $(0, \pi)$, we have that

$$
P\left(\phi_{0}\right)=-\phi_{0}+\pi
$$

In terms of $x$ and $t$ the parity transformation (3.49) takes the form of (3.21) with

$$
\begin{aligned}
x_{\Delta} & =\frac{\eta_{1} \eta_{2}}{\sinh \left(\alpha_{1}-\alpha_{2}\right)}\left[\eta_{2} \sinh \alpha_{2}\left(\delta-\eta_{1} x_{0}^{(1)} \cosh \alpha_{1}\right)-\eta_{1} \sinh \alpha_{1}\left(\delta-\eta_{2} x_{0}^{(2)} \cosh \alpha_{2}\right)\right], \\
t_{\Delta} & =\frac{\eta_{1} \eta_{2}}{\sinh \left(\alpha_{1}-\alpha_{2}\right)}\left[\delta \eta_{2} \cosh \alpha_{2}-\eta_{1} \cosh \alpha_{1}\left(\eta_{2}\left(x_{0}^{(1)}-x_{0}^{(2)}\right) \cosh \alpha_{2}+\delta\right)\right] .
\end{aligned}
$$


From (3.50) we note that the even and odd components of $\phi_{0}$ are given by

$$
\phi_{0}^{( \pm)} \equiv \frac{1}{2}(1 \pm P)\left(\phi_{0}\right), \quad \text { and so } \quad \phi_{0}^{(-)}=\phi_{0}-\frac{\pi}{2} ; \quad \phi_{0}^{(+)}=\frac{\pi}{2}
$$

Note also that $\sin \left(4 \phi_{0}\right)=\sin \left(4 \phi_{0}^{(-)}\right)$and so we see that $\phi_{0}^{(-)}$and $\phi_{0}^{(+)}$are both solutions of the sine-Gordon equation, i.e.

$$
\partial^{2} \phi_{0}^{( \pm)}+\frac{1}{4} \sin \left(4 \phi_{0}^{( \pm)}\right)=0
$$

In fact, $\phi_{0}^{(+)}$is a vacuum solution and $\phi_{0}^{(-)}$is a proper two-soliton solution (with $\phi_{0}$ being given by (3.41)).

\subsubsection{The breather solutions}

The moving breather solution of the sine-Gordon equation (3.40) is also obtained from a tau function, through (3.41), given by [7]

$$
\tau_{0}=1+e^{2 \Gamma_{R}}+2 i(\operatorname{cotan} \theta) e^{\Gamma_{R}} \sin \Gamma_{I},
$$

with

$$
\Gamma_{R}=\frac{\cos \theta}{\sqrt{1-v^{2}}}(x-v t), \quad \Gamma_{I}=\frac{\sin \theta}{\sqrt{1-v^{2}}}(t-v x) .
$$

So, from (3.41) one gets that

$$
\phi_{0}=\operatorname{ArcTan} \frac{(\operatorname{cotan} \theta) \sin \Gamma_{I}}{\cosh \Gamma_{R}},
$$

where $v$ is the speed of the breather, and $\sin \theta$ is its frequency (in its rest frame). Note that the argument of ArcTan in (3.56) never diverges and so $\phi_{0}$ never passes through $\pm \frac{\pi}{2}$. Thus, for the breather solution it is more convenient to take the domain of ArcTan to be $\left(-\frac{\pi}{2}, \frac{\pi}{2}\right)$. We now introduce the space-time parity transformation

$$
P: \quad(x, t) \rightarrow(-x,-t)
$$

and comparing it with (3.21) we see that in this case $x_{\Delta}=t_{\Delta}=0$. Under this parity transformation, the breather solution (3.56) is odd, i.e.

$$
P\left(\phi_{0}\right)=-\phi_{0}
$$

So, in the breather case we see that

$$
\phi_{0}^{( \pm)} \equiv \frac{1}{2}(1 \pm P)\left(\phi_{0}\right) \quad \rightarrow \quad \phi_{0}^{(-)}=\phi_{0} ; \quad \phi_{0}^{(+)}=0
$$

and so $\phi_{0}^{(-)}$and $\phi_{0}^{(+)}$are again both solutions of the sine-Gordon equation (3.40), with the first being the breather itself and the second a trivial vacuum solution. 


\subsubsection{The parity versus dynamics argument for our models}

We start by observing that the sine-Gordon potential (3.6) is invariant under (3.50) and (3.58). Therefore, the sine-Gordon theory satisfies the requirements (3.37) and (3.38), for quasi-integrability, within the two-soliton and breather sector, as it should, since it is an exact integrable field theory. We now discuss the conditions which the theories, which are smooth deformations of the sine-Gordon, should satisfy to be called quasi-integrable.

We shall restrict our attention to the theories of the previous section; i.e. to theories with equations of motion given by (3.5), where the potential $V$ is a smooth deformation of the sine-Gordon potential (3.6), in the sense that $V$ depends upon a parameter $\varepsilon$ such that for $\varepsilon=0, V$ becomes (3.6). In order to perform the expansion around the sine-Gordon model we shall work with two-soliton and breather solutions which are odd under the parity. We will also expand the field $\phi$, as a solution of (3.5), in a power series in $\varepsilon$, i.e.

$$
\phi=\phi_{0}^{(-)}+\varepsilon \phi_{1}+\varepsilon^{2} \phi_{2}+\ldots
$$

Thus, the potential $V$ depends upon $\varepsilon$ explicitly, and also implicitly through $\phi$. Then the Taylor expansion around $\varepsilon=0$ of its first derivative w.r.t. $\phi$ takes the form

$$
\begin{aligned}
\frac{\partial V}{\partial \phi}= & \left.\frac{\partial V}{\partial \phi}\right|_{\varepsilon=0}+\left[\frac{d}{d \varepsilon}\left(\frac{\partial V}{\partial \phi}\right)\right]_{\varepsilon=0} \varepsilon+\ldots \\
= & \left.\frac{\partial V}{\partial \phi}\right|_{\varepsilon=0}+\left[\frac{\partial^{2} V}{\partial \varepsilon \partial \phi}+\frac{\partial^{2} V}{\partial \phi^{2}} \frac{\partial \phi}{\partial \varepsilon}\right]_{\varepsilon=0} \varepsilon \\
& +\frac{1}{2}\left[\frac{\partial^{3} V}{\partial \varepsilon^{2} \partial \phi}+2 \frac{\partial^{3} V}{\partial \varepsilon \partial \phi^{2}} \frac{\partial \phi}{\partial \varepsilon}+\frac{\partial^{2} V}{\partial \phi^{2}} \frac{\partial^{2} \phi}{\partial \varepsilon^{2}}+\frac{\partial^{3} V}{\partial \phi^{3}}\left(\frac{\partial \phi}{\partial \varepsilon}\right)^{2}\right]_{\varepsilon=0} \varepsilon^{2}+\ldots
\end{aligned}
$$

In consequence, splitting (3.5) in powers of $\varepsilon$ we find that $\phi_{0}$ satisfies the sine-Gordon equation (3.40). The higher components of the field $\phi$ satisfy

$$
\begin{aligned}
\partial^{2} \phi_{1}+\left.\frac{\partial^{2} V}{\partial \phi^{2}}\right|_{\varepsilon=0} \phi_{1}= & -\left.\frac{\partial^{2} V}{\partial \varepsilon \partial \phi}\right|_{\varepsilon=0} \\
\partial^{2} \phi_{2}+\left.\frac{\partial^{2} V}{\partial \phi^{2}}\right|_{\varepsilon=0} \phi_{2}= & -\frac{1}{2}\left[\left.\frac{\partial^{3} V}{\partial \varepsilon^{2} \partial \phi}\right|_{\varepsilon=0}+\left.2 \frac{\partial^{3} V}{\partial \varepsilon \partial \phi^{2}}\right|_{\varepsilon=0} \phi_{1}+\left.\frac{\partial^{3} V}{\partial \phi^{3}}\right|_{\varepsilon=0} \phi_{1}^{2}\right] \\
\partial^{2} \phi_{3}+\left.\frac{\partial^{2} V}{\partial \phi^{2}}\right|_{\varepsilon=0} \phi_{3}= & -\frac{1}{3 !}\left[\left.\frac{\partial^{4} V}{\partial \varepsilon^{3} \partial \phi}\right|_{\varepsilon=0}+\left.3 \frac{\partial^{4} V}{\partial \varepsilon^{2} \partial \phi^{2}}\right|_{\varepsilon=0} \phi_{1}+\left.6 \frac{\partial^{3} V}{\partial \varepsilon \partial \phi^{2}}\right|_{\varepsilon=0} \phi_{2}\right. \\
& \left.+\left.3 \frac{\partial^{4} V}{\partial \varepsilon \partial \phi^{3}}\right|_{\varepsilon=0} \phi_{1}^{2}+\left.6 \frac{\partial^{3} V}{\partial \phi^{3}}\right|_{\varepsilon=0} \phi_{1} \phi_{2}+\left.\frac{\partial^{4} V}{\partial \phi^{4}}\right|_{\varepsilon=0} \phi_{1}^{3}\right]
\end{aligned}
$$

and so on.

We can write all these equations, generically, as

$$
\partial^{2} \phi_{n}+\left.\frac{\partial^{2} V}{\partial \phi^{2}}\right|_{\varepsilon=0} \phi_{n}=f_{n} \quad n=1,2,3, \ldots
$$

Note that if one expands $V$ in powers of $\varepsilon$, taking into account only its explicit dependence on $\varepsilon$ and neglecting its implicit dependence on $\varepsilon$ through $\phi$, one gets that

$$
V=\left.V\right|_{\varepsilon=0}+\left.\varepsilon \frac{\partial V}{\partial \varepsilon}\right|_{\varepsilon=0}+\left.\frac{\varepsilon^{2}}{2} \frac{\partial^{2} V}{\partial \varepsilon^{2}}\right|_{\varepsilon=0}+\ldots
$$


where $\left.V\right|_{\varepsilon=0}$ is the sine-Gordon potential (3.6). Thus, one finds that

$$
\left.\frac{\partial^{2} V}{\partial \phi^{2}}\right|_{\varepsilon=0}=\left.\frac{\partial^{2}\left(\left.V\right|_{\varepsilon=0}\right)}{\partial \phi^{2}}\right|_{\varepsilon=0}=\cos \left(4 \phi_{0}^{(-)}\right) .
$$

Indeed, one can check explicitly that the potentials (2.10), (2.13) and (2.16) mentioned in the last section do satisfy (3.67). In fact this is true in all models which involve deformations as discussed in [6] as for all of them $\left.\frac{\partial^{2}\left(\left.V\right|_{\varepsilon=0}\right)}{\partial \phi^{2}}\right|_{\varepsilon=0}=\cos \left(4 \phi_{0}\right)$. Thus the approach of [6] guarantees (3.67).

We now split (3.65) into the even and odd parts under the parity $P$, as

$$
\partial^{2} \phi_{n}^{( \pm)}+\cos \left(4 \phi_{0}^{(-)}\right) \phi_{n}^{( \pm)}=f_{n}^{( \pm)} \quad \text { with } \quad \star^{( \pm)} \equiv \frac{1}{2}(1 \pm P) \star
$$

Then using (2.10), (2.13) and (2.16) one can verify that

$$
\begin{aligned}
f_{1}^{(1)} \equiv & -\left.\frac{\partial^{2} V_{1}}{\partial \varepsilon \partial \phi}\right|_{\varepsilon=0} \\
= & \frac{1}{16}\left[\left(\pi^{2}-12\left(\phi_{0}^{(-)}\right)^{2}\right) \sin \left(4 \phi_{0}^{(-)}\right)+4\left(4\left(\phi_{0}^{(-)}\right)^{2}-\pi^{2}+3\right) \phi_{0}^{(-)} \cos \left(4 \phi_{0}^{(-)}\right)-12 \phi_{0}^{(-)}\right], \\
f_{1}^{(2)} \equiv & -\left.\frac{\partial^{2} V_{2}}{\partial \varepsilon \partial \phi}\right|_{\varepsilon=0} \\
= & \frac{1}{8}\left[\left(-8\left(\phi_{0}^{(-)}\right)^{2}+4 \pi \phi_{0}^{(-)}-2\right) \cos \left(4 \phi_{0}^{(-)}\right)-\left(\pi-4 \phi_{0}^{(-)}\right) \sin \left(4 \phi_{0}^{(-)}\right)+2\right] \\
f_{1}^{(3)} \equiv & -\left.\frac{\partial^{2} V_{3}}{\partial \varepsilon \partial \phi}\right|_{\varepsilon=0} \\
= & \frac{1}{32}\left[\left(16 \gamma \phi_{0}^{(-)}-4 \pi \gamma-12\left(\phi_{0}^{(-)}\right)^{2}+\pi^{2}\right) \sin \left(4 \phi_{0}^{(-)}\right)+8 \gamma-12 \phi_{0}^{(-)}\right. \\
& \left.-4\left(\gamma\left(8\left(\phi_{0}^{(-)}\right)^{2}-4 \pi \phi_{0}^{(-)}+2\right)+\phi_{0}^{(-)}\left(-4\left(\phi_{0}^{(-)}\right)^{2}+\pi^{2}-3\right)\right) \cos \left(4 \phi_{0}^{(-)}\right)\right] .
\end{aligned}
$$

Then we note that $\frac{1}{2}(1-P) f_{1}^{(j)} \neq 0$, for $j=1,2,3$, and that

$$
\begin{aligned}
& \frac{1}{2}(1+P) f_{1}^{(1)}=0, \\
& \frac{1}{2}(1+P) f_{1}^{(2)} \neq 0, \\
& \frac{1}{2}(1+P) f_{1}^{(3)}=\left\{\begin{array}{ccc}
0 & \text { for } & \gamma=0, \\
\neq 0 & \text { for } & \gamma \neq 0 .
\end{array}\right.
\end{aligned}
$$

Thus we see that for the theories described by the potential $V_{1}$, given in (2.10), and the potential $V_{3}$, given in (2.16), for $\gamma=0$, the even part (under the parity) of the first order field $\phi_{1}$ satisfies homogeneous equations, and the odd part of the first order field $\phi_{1}$ satisfies non-homogeneous equations, i.e.

$$
\begin{aligned}
& \partial^{2} \phi_{1}^{(+)}+\left.\frac{\partial^{2} V}{\partial \phi^{2}}\right|_{\varepsilon=0} \phi_{1}^{(+)}=0, \\
& \partial^{2} \phi_{1}^{(-)}+\left.\frac{\partial^{2} V}{\partial \phi^{2}}\right|_{\varepsilon=0} \phi_{1}^{(-)} \neq 0 \quad \text { for } \quad V_{1} \text { and }\left.V_{3}\right|_{\gamma=0} .
\end{aligned}
$$


Thus, for such cases, one can always choose $\phi_{1}^{(+)}=0$. Indeed, if $\phi_{1}$ is a solution, so is $\phi_{1}=\phi_{1}-\phi_{1}^{(+)}=\phi_{1}^{(-)}$.

One can also check that if one chooses $\phi_{1}^{(+)}=0$, then it follows that for the theories governed by the potentials $V_{1}$ and $\left.V_{3}\right|_{\gamma=0}$, the second order field $\phi_{2}$ satisfies properties similar to those of $\phi_{1}$, i.e. its even part under the parity $\phi_{2}^{(+)}$, satisfies homogenous equations, and its odd part $\phi_{2}^{(-)}$, satisfies non-homogeneous equations. So, one can similarly take $\phi_{2}^{(+)}=0$.

Continuing this process one reaches the conclusion that it is always possible to have two-soliton-like and breather-like solutions for the theories with potentials $V_{1}$ and $\left.V_{3}\right|_{\gamma=0}$, which are odd under the parity $P$. In addition, one can check that when evaluated on such odd solutions the potentials $V_{1}$ and $\left.V_{3}\right|_{\gamma=0}$ are even under the parity $P$. Therefore, such theories satisfy the requirements (3.37) and (3.38) and so they possess an infinite number of charges satisfying the property (3.36), i.e. such theories are, using our definition, quasi-integrable.

The same argument does not apply to the theories governed by the potentials $V_{2}$ and $\left.V_{3}\right|_{\gamma \neq 0}$, and so they cannot be considered quasi-integrable. Of course, it still may be the case, that they satisfy (3.39) for other reasons.

\section{Numerical simulations}

To perform the numerical simulations to check our hypothesis we still have to construct two-soliton configurations in our models.

In our numerical simulations we want to analyze the quasi-conservation of the first non-trivial charge beyond the energy itself, i.e. the charge $Q^{(3)}$ defined in (3.18). We do this by computing the corresponding anomaly $\alpha^{(3)}$ also introduced in (3.18). One can check that the factor $\gamma^{(3)}$ entering in the definition of $\alpha^{(3)}$ is given by (see [1])

$$
\gamma^{(3)}=i \omega \partial_{-}^{2} \phi
$$

with $\partial_{-}=\partial_{t}-\partial_{x}$. Thus, using (3.18) and (3.4), one finds that

$$
\alpha^{(3)}=-8 \int_{-\infty}^{\infty} d x \partial_{-} \phi \partial_{-}^{2} \phi\left[\frac{d^{2} V}{d \phi^{2}}+16 V-1\right],
$$

where we have chosen $\omega=4$ and $m=1$, which are the values that make $X$ vanish when $V$ is the sine-Gordon potential (see comments around (3.6)). We also want to compute the so-called integrated anomaly given by (see (3.17))

$$
\beta^{(3)}=-\frac{1}{2} \int_{t_{0}}^{t} d t^{\prime} \alpha^{(3)}=Q^{(3)}(t)-Q^{(3)}\left(t_{0}\right),
$$

where $t_{0}$ is the initial time of the simulation, usually taken to be zero.

It is easy to check for the potentials of the form (2.6) one has that

$$
\frac{d^{2} V}{d \phi^{2}}+16 V-1=2\left[\frac{\partial}{\partial \phi}\left(G \frac{\partial G}{\partial \phi}\right)+2 G^{2}-2\right] V_{0}+3 \frac{d V_{0}}{d \psi} \frac{\partial G}{\partial \phi},
$$

where $V_{0}=\sin ^{2}(\psi(\phi))$ and $G=\frac{\partial \phi}{\partial \psi}$.

Note that in the case of the sine-Gordon potential, which corresponds to $\psi(\phi)=\phi$ and so $G=1$, one sees that this quantity indeed vanishes. 


\subsection{General considerations}

Our numerical simulations were performed using the 4th order Runge-Kutta method of simulating time evolution. We experimented with various grid sizes and numbers of points and most simulations were performed on lattices of 10001 lattice points with lattice spacing of 0.01 (so they covered the region of $(-50.0,50.0)$. Time step $d t$ was 0.0001 . The solitons (for the soliton-soliton scattering) were placed at \pm 35.00 and stretched \pm 5.00 from their positions hence at the edges of the grid the fields resembled the vacuum configurations which were modified only by waves that were emitted during the scattering. The same was true for the breather-like structures - except that this time the field configurations were different from their vacuum values only for a very small range of $x$ (i.e. for $|x|<20$ ).

At the edges of the grid (i.e. for $49.50<|x|<50.00$ ) we absorbed the waves reaching this region (by decreasing the time change of the magnitude of the field there).

In consequences, the total energy was not conserved but the only energy which was absorbed was the energy of radiation waves. Hence the total energy was effectively the energy of the field configuration which we wanted to study.

As we will discuss later in this section the energy was very well conserved in all solitonsoliton scatterings and much less so for the evolution of our breather-like structures.

\subsection{Two soliton configurations}

For the first model we note that we can put one soliton $\psi$ to lie between $\left(-\frac{\pi}{2}, 0\right)$ and the second one to lie between $\left(0, \frac{\pi}{2}\right)$. Thus our initial condition is given by ( $f$ stands for the initial field configuration)

$$
f=\frac{g}{1+\varepsilon \frac{\pi^{2}}{4}-\varepsilon g^{2}}
$$

where

$$
g=-\operatorname{ArcTan}\left[\exp \left(\frac{-\left(x+a_{1}\right)+v t}{\sqrt{1-v^{2}}}\right)\right] \text { for } \quad x<0
$$

and

$$
g=\operatorname{ArcTan}\left[\exp \left(\frac{x-a_{1}+v t}{\sqrt{1-v^{2}}}\right)\right] \text { for } \quad x>0,
$$

and where $a_{1} \gg 0$.

In this case one soliton is placed at $-a_{1}$ and the other one at $a_{1}$. For $x<0 f(x, 0)$ varies from $-\frac{\pi}{2}$ (for $x \sim-\infty$ ) to $f \sim 0$ for $x=0$. For $x>0 f(x, 0)$ varies from 0 (for $x \sim 0$ ) to $f=\frac{\pi}{2}$ for $x \sim \infty$.

In the second case we take

$$
f=\frac{\left(1+\varepsilon \frac{\pi}{2}\right) g}{1+\varepsilon g}
$$

where

$$
g=\operatorname{ArcTan}\left[\exp \left(\frac{\left(x+a_{1}\right)-v t}{\sqrt{1-v^{2}}}\right)\right] \quad \text { for } \quad x<0
$$

and

$$
g=\operatorname{ArcTan}\left[\exp \left(\frac{x-a_{1}+v t}{\sqrt{1-v^{2}}}\right)\right]+\frac{\pi}{2} \quad \text { for } \quad x>0,
$$

and where again $a_{1} \gg 0$. 

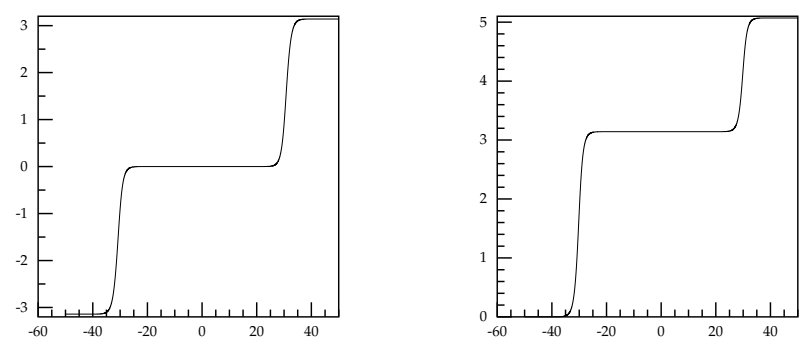

Figure 4. The initial fields of two solitons for $\varepsilon=0.1, v=0.05$. On the left we show the initial configuration (4.5) (rescaled by a factor 2) for the first class of model defined in section 2.1. On the right we show the initial configuration (4.8) (also rescaled by a factor 2) for the second class of models defined in section 2.2 .
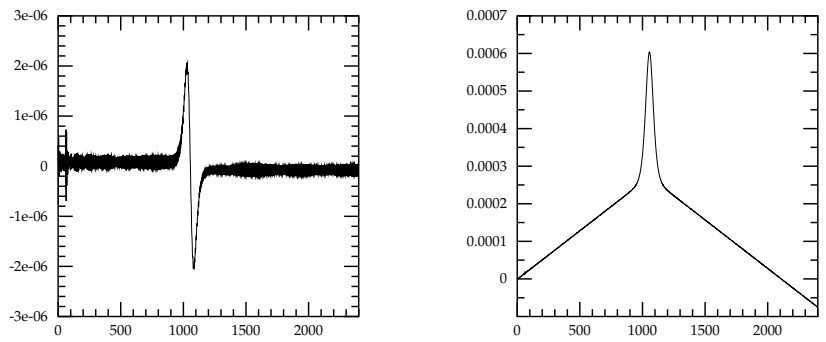

Figure 5. Anomalies for a two-soliton solution of the first class of models, defined in section 2.1, for $\varepsilon=0.01$ and $v=0.025$. On the left we show the anomaly $\alpha^{(3)}$ given in (4.2), and on the right the time integrated anomaly $\beta^{(3)}$ given in (4.3).

The two solitons are again placed at $\pm a_{1}$ but this time for $x<0 f(x, 0)$ varies from 0 $($ for $x \sim-\infty)$ to $f \sim \frac{\pi}{2}$ for $x=0$. For $x>0 f(x, 0)$ varies from $\frac{\pi}{2}$ for $x \sim 0$ to $f \sim \frac{\pi\left(1+\varepsilon \frac{\pi}{2}\right)}{1+\varepsilon \pi}$ for $x \sim \infty$.

In figure 4 we plot the fields at $t=0$ used in our simulations. The values of $\varepsilon, a_{1}$ and $v$ are $\varepsilon=0.1 a_{1}=30.0$ and $v=0.05$.

We note that the first model possesses the required symmetry while the second one does not.

Next we have performed simulations of the scatterings and the anomalies in each model for many values of all parameters.

Below we present some of our results. 

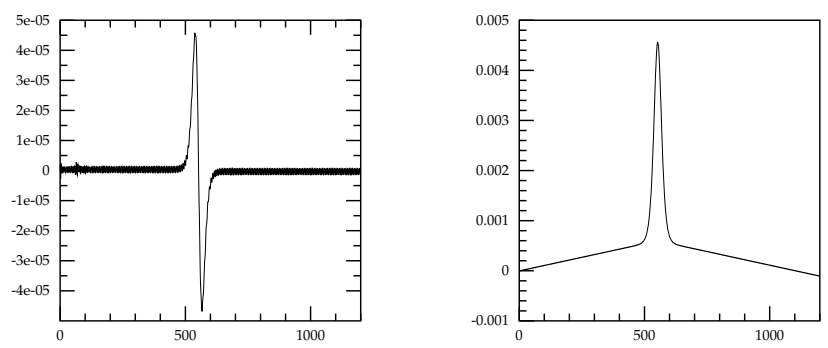

Figure 6. Anomalies for a two-soliton solution of the first class of models, defined in section 2.1, for $\varepsilon=0.1$ and $v=0.05$. On the left we show the anomaly $\alpha^{(3)}$ given in (4.2), and on the right the time integrated anomaly $\beta^{(3)}$ given in (4.3).
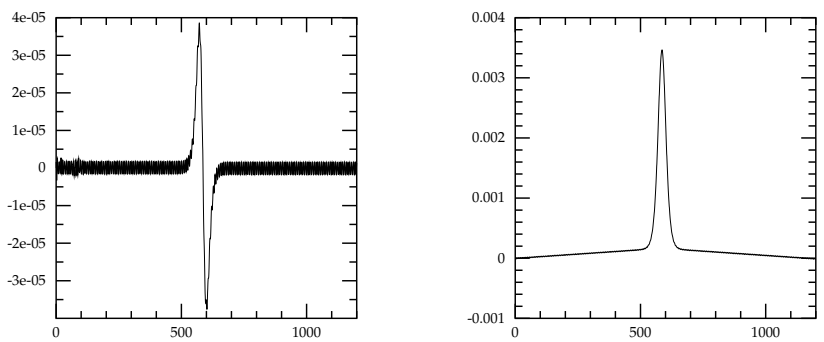

Figure 7. Anomalies for a two-soliton solution of the first class of models, defined in section 2.1, for $\varepsilon=1.0$ and $v=0.05$. On the left we show the anomaly $\alpha^{(3)}$ given in (4.2), and on the right the time integrated anomaly $\beta^{(3)}$ given in (4.3).

First we present the results in the first model. In figures $5,6,7$ we present the expressions of the anomaly (and time integrated) for 3 values of $\varepsilon(\varepsilon=0.01$ in figure 5 , $\varepsilon=0.1$ in figure 6 and $\varepsilon=1.0$ in figure 7 ) The left-hand plots present the time dependence of anomaly; while the plots to the right of them show the time integrated anomaly. We note that the unintegrated anomaly increases as the solitons come together, then vanishes when the solitons reflect and changes sign when they are moving away from each other. This change is responsible for the almost complete cancelation of the total anomaly and so is 'generates' quasi-integrability.

Then we performed the simulation of the models of the second class. The results are presented in figures 8, 9 and 10. We note that this time the nonintegrated anomaly behaves very differently. Not only it stayed of only one sign but is in fact maximal when the two 

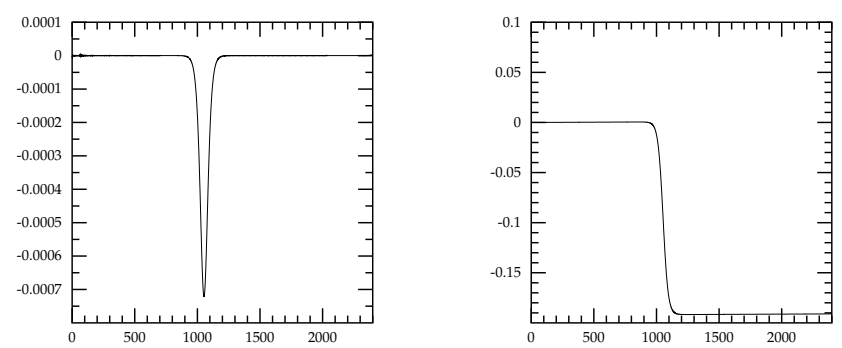

Figure 8. Anomalies for a two-soliton solution of the second class of models, defined in section 2.2, for $\varepsilon=0.01$ and $v=0.025$. On the left we show the anomaly $\alpha^{(3)}$ given in (4.2), and on the right the time integrated anomaly $\beta^{(3)}$ given in (4.3).
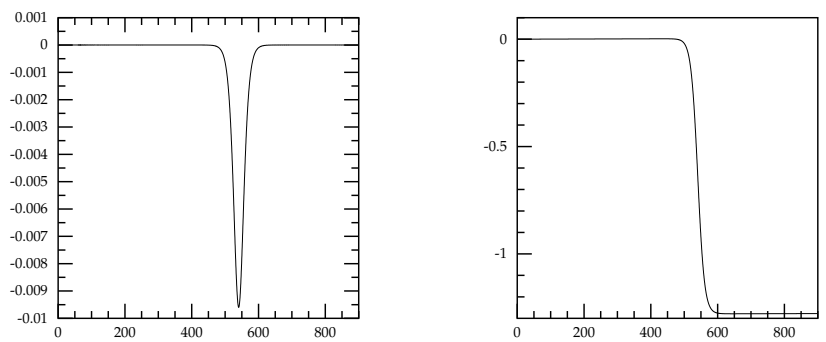

Figure 9. Anomalies for a two-soliton solution of the second class of models, defined in section 2.2, for $\varepsilon=0.1$ and $v=0.05$. On the left we show the anomaly $\alpha^{(3)}$ given in (4.2), and on the right the time integrated anomaly $\beta^{(3)}$ given in (4.3).

solitons come to rest before reflecting. Hence, the integrated anomaly changes dramatically when the two solitons are very close together.

To be completely certain of our results we have also performed a further set of simulations based on a different approach. In the simulations discussed so far we have put two moving solitons some distance apart and built our initial conditions based on such a superposition. However, even for solitons quite far apart (and they are very localized) their superposition has a very small error at the place where they overlap, namely around $x=0$ in our case. So to verify that this error was not responsible for the difference in the anomaly behaviour in the two cases, we have also used a different form of the initial condition; namely we based it on a well known two soliton solution of the Sine Gordon model. We took such a solution of the initial $\psi$ and calculated $\phi$ and $\frac{\partial \phi}{\partial t}$ from it. 

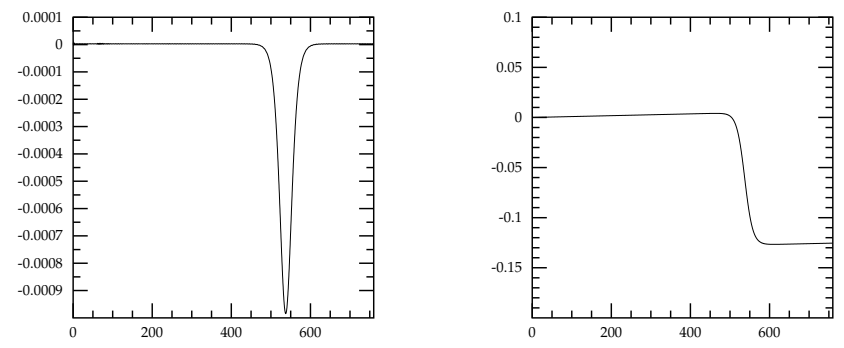

Figure 10. Anomalies for a two-soliton solution of the second class of models, defined in section 2.2, for $\varepsilon=1.0$ and $v=0.05$. On the left we show the anomaly $\alpha^{(3)}$ given in (4.2), and on the right the time integrated anomaly $\beta^{(3)}$ given in (4.3).

Then we performed the simulations once again and compared the obtained results with those found before. The agreement was surprisingly good. We do not present here any files as the two sets of files are 'almost' indistinguishable from each other. This suggest to us that the observed behaviour is a genuine effect and not generated by any 'numerical artifacts'.

To study in detail how this change takes place we looked at the problem in the third class of models. We then calculated the anomalies in the third class models and performed many simulations for many values of $\varepsilon$ and $\gamma$. For most values the results were qualitatively the same as in the previous models but for ranges of parameters $\varepsilon$ and $\gamma$ we observed the transfer of behaviour from the model with approximate symmetry to the one in which this symmetry is broken. In figures $11-15$ we illustrate this transfer. As we increase the values of $\gamma$ the unintegrated anomaly becomes asymmetric leading to the change of total anomaly. Figures 12 and 13 show that this symmetry can be broken in either direction (depending on the sign of $\gamma$ ).

Again we repeated the simulations with two sets of initial conditions and we have found the results being essentially the same. As is clear from the figures (see figures 11-15) at small values of $\gamma$ the results resemble the results of the first model and as we increase $\gamma$ (in magnitude) they gradually change to those of the second class model. The change smoothly interpolates between the two behaviours. This supports our belief that the results are not numerical artifacts but present the genuine behaviour of the system.

One may wonder how it is possible that $\beta^{(3)}$ is nonzero in the second model (or in the third model for $\gamma \neq 0$ ) as asymptotically solitons do not influence each other and for an isolated soliton anomaly vanishes. One may even wonder whether the behaviour of the anomaly (as shown in figures 8-15) is not due to numerical errors.

However, the solitons do interact when they are close together and this interaction can and does change some of their properties as we have discovered by looking at this problem more closely. This was done by looking at two solitons in the third class of models for several values of $\gamma$ close to zero. The two solitons were sent towards each 

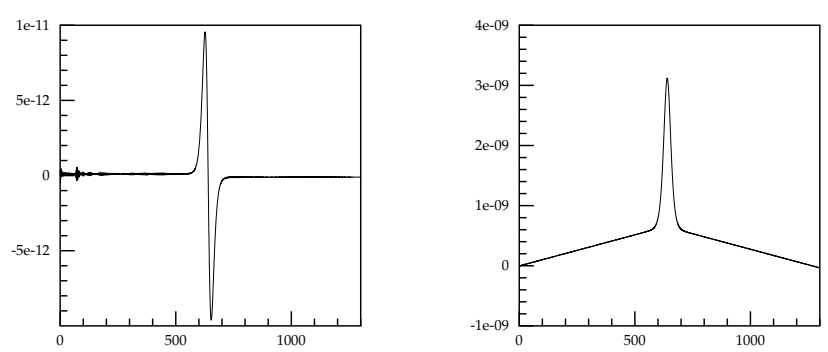

Figure 11. Anomalies for a two-soliton solution of the third class of models, defined in section 2.3, for $\varepsilon=1 \times 10^{-8}, v=0.05$ and $\gamma=1 . \times 10^{-5}$. On the left we show the anomaly $\alpha^{(3)}$ given in (4.2), and on the right the time integrated anomaly $\beta^{(3)}$ given in (4.3).
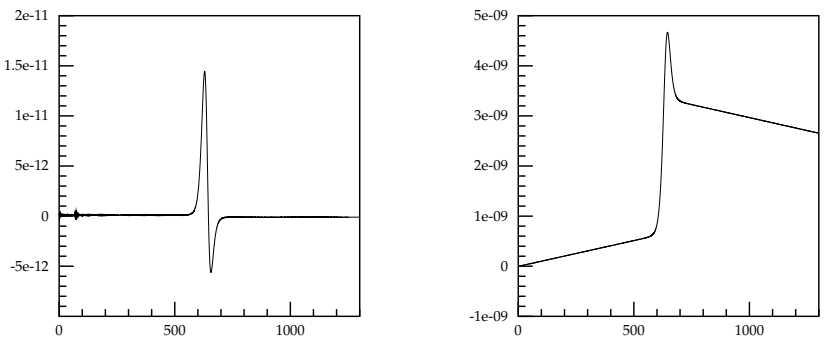

Figure 12. Anomalies for a two-soliton solution of the third class of models, defined in section 2.3, for $\varepsilon=1 \times 10^{-8}, v=0.05$ and $\gamma=-0.002$. On the left we show the anomaly $\alpha^{(3)}$ given in (4.2), and on the right the time integrated anomaly $\beta^{(3)}$ given in (4.3).

other with the same velocity and we looked at their individual energies and charges $Q^{(3)}$ during the scattering. Of course when the solitons were close together these quantities were not very well defined; so we looked at their approximate values by computing separately their energies and charges $Q^{(3)}$ by integrating their densities on the left side of the grid, corresponding to $x<0$, and on the right side of the grid corresponding to $x \geq 0$.

In figures 16-17 we present results seen in a typical simulation (corresponding to $\gamma=0.009$, and $\varepsilon=0.3$ ). Figure 16 shows (from left to right) the time dependence of the total energy, the time dependence of the energy on the right side of the grid $(x \geq 0)$, and finally the energy on the left side of the grid $(x<0)$. We see that the total energy is very well conserved but the contributions from the two sides of the grid change during the scattering. Hence the two scattered solitons are different from the two incoming ones (they move with different speeds and are slightly perturbed). 

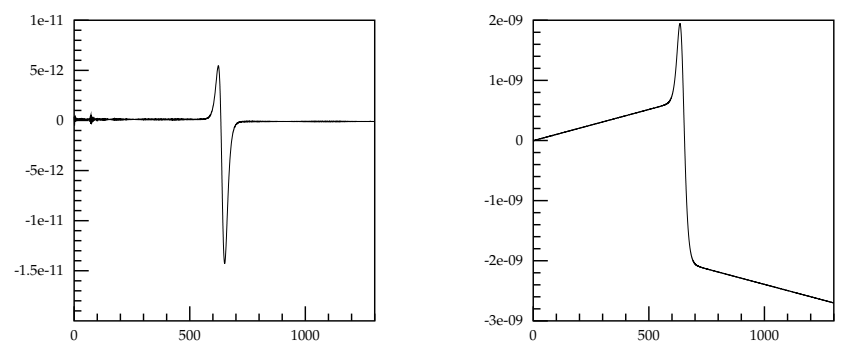

Figure 13. Anomalies for a two-soliton solution of the third class of models, defined in section 2.3, for $\varepsilon=1 \times 10^{-8}, v=0.05$ and $\gamma=0.002$. On the left we show the anomaly $\alpha^{(3)}$ given in (4.2), and on the right the time integrated anomaly $\beta^{(3)}$ given in (4.3).
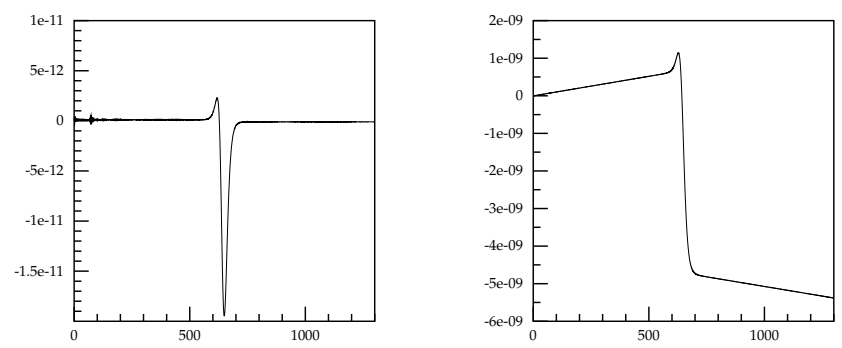

Figure 14. Anomalies for a two-soliton solution of the third class of models, defined in section 2.3, for $\varepsilon=1 \times 10^{-8}, v=0.05$ and $\gamma=0.004$. On the left we show the anomaly $\alpha^{(3)}$ given in (4.2), and on the right the time integrated anomaly $\beta^{(3)}$ given in (4.3).

Figure 17 presents the similar results for the time evolution of $Q^{(3)}$. This time we see, however, that the changes are non-symmetrical and that they result in the change of value of $Q^{(3)}$. This explains our results on the anomaly; the results shown here are consistent with what we have seen in our studies of the anomaly. Of course, our numerical results do have small numerical errors. Luckily, they are much smaller than the effects described above. This we have verified by performing many similar simulations varying time step and $x$ step and by varying the starting files (varying their parameters and by either 'sewing' two solitons as described in section 4.1 or by taking an exact two soliton configuration of the Sine Gordon model and then mapping it into a field from which the initial conditions were calculated). All these numerical simulations gave us essentially the same results demonstrating the reliability of our claims. They have also shown that to go further we 

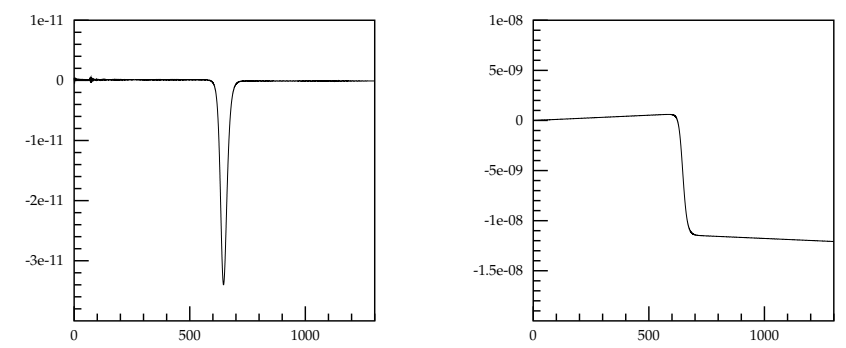

Figure 15. Anomalies for a two-soliton solution of the third class of models, defined in section 2.3, for $\varepsilon=1 \times 10^{-8}, v=0.05$ and $\gamma=0.009$. On the left we show the anomaly $\alpha^{(3)}$ given in (4.2), and on the right the time integrated anomaly $\beta^{(3)}$ given in (4.3).
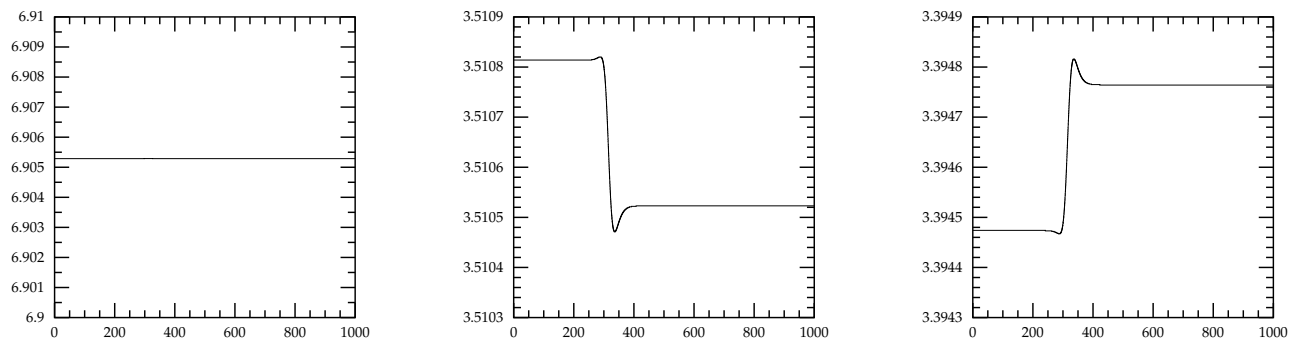

Figure 16. A study of the behavior of the energy in the scattering of two solitons of the third class of models defined in section 2.3, for $\gamma=0.009$, and $\varepsilon=0.3$. In the left plot we show the total energy of the two soliton configuration as a function of time. In the center plot we show the energy in the right side of the grid $(x \geq 0)$ as a function of time. Finally in the right plot we show the energy in the left side of the grid $(x<0)$ as a function of time.

have to look at situations in which solitons interact with each other as often as possible and this suggests looking at breather-like configurations involving one soliton and one antisoliton. Hence in the next section we report our preliminary results on such configurations.

\subsection{Breather-like configurations}

In all two soliton configurations the interactions between the two solitons were very shortlived; the solitons interacted with each other only when they were very closed to each other and after the reflection their interactions rapidly decreased. Our numerical results show this very clearly; anomaly changes significantly when the solitons are close together. 

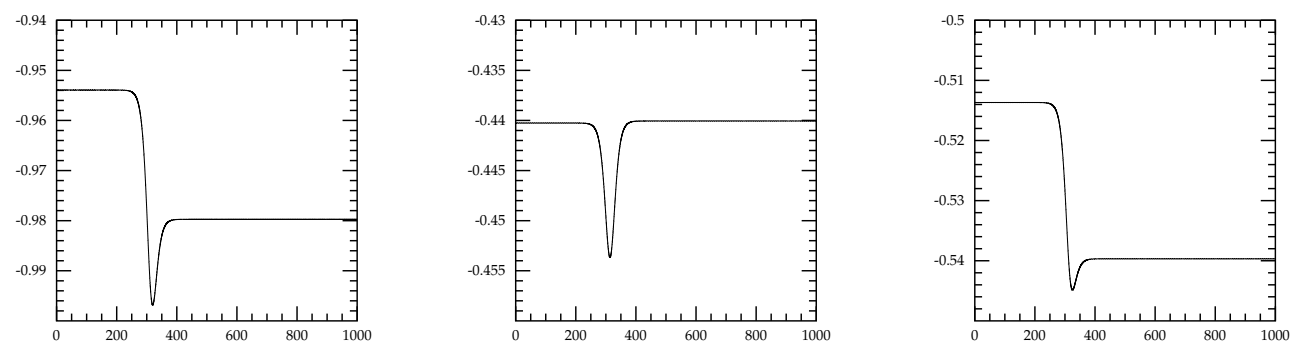

Figure 17. A study of the behavior of the charge $Q^{(3)}$, defined in (3.18), in the scattering of two solitons of the third class of models defined in section 2.3 , for $\gamma=0.009$, and $\varepsilon=0.3$. In the left plot we show the total charge $Q^{(3)}$ of the two soliton configuration as a function of time. In the center plot we show the amount of $Q^{(3)}$ in the right side of the grid $(x \geq 0)$ as a function of time. Finally in the right plot we show the amount of $Q^{(3)}$ in the left side of the grid $(x<0)$ as a function of time.

On the other hand when we consider breather-like configurations (i.e. trapped pairs of a soliton and an antisoliton) they constantly interact with each other and so the anomalies change all the time. Whether their behaviour is consistent with our anomaly behaviour for breather like objects (3.36) can be studied numerically too and we have performed several simulations in the third class of models.

To start the simulations we took a breather configuration for the sine-Gordon model (3.56) and then perfomed the change of variables (2.14) to obtain the corresponding $\phi$ field. We then used this field and its derivative at $t=0$ as the initial conditions for the simulations.

We have performed several simulations for different values of the frequency of the breather i.e. $\nu$ in (3.56) and for various values of $\varepsilon$ and $\gamma$.

Most of our simulations were performed for $\nu=0.5$. These simulations have shown that, indeed, for $\gamma=0.00$ we have quasi-integrability, i.e. the behaviour is consistent with (3.36). The anomaly does change all the time and even the time integrated anomaly does depend on time. However, the oscillations are approximately around a fixed value and so, shifting in time and choosing $t_{\Delta}$ appropriately we see that (3.36) holds. When we take small $\gamma$ the effects are not very different (i.e. one should wait long enough to see the difference but for larger values of $\gamma$ the effects are clear as the time integrated anomaly decreases. This is true for positive and negative values of $\gamma \neq 0$. In figure 18, 19 and 20 we present results of our simulations for $\nu=0.5$ for $\epsilon=0.1$ and three values of $\gamma$, namely $\gamma=$ -0.5 (figure 18), $\gamma=0.0$ (figure 19) and $\gamma=0.5$ in figure 20. The left hand plots in each set of pictures show unintegrated anomaly, the right hand sides ones the time integrated ones.

The presented results clearly show that the $\gamma=0$ case is somewhat special as then the time integrated anomaly exibits the expected symmetry principle (3.36). 

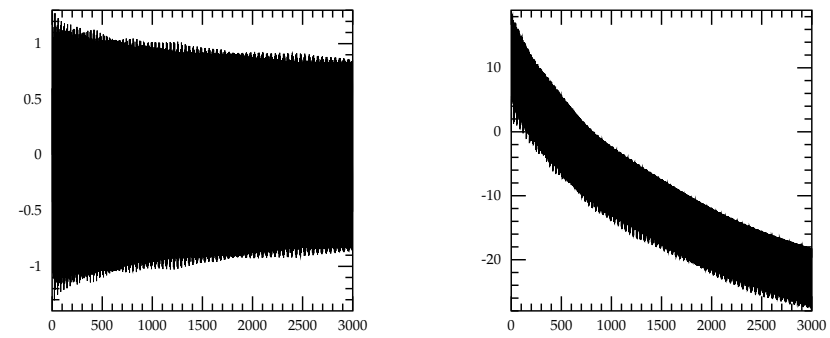

Figure 18. Anomalies for a breather-like configuration of the third class of models, defined in section 2.3 , for $\varepsilon=0.1$ and $\gamma=-0.5$. On the left we show the anomaly $\alpha^{(3)}$ given in (4.2), and on the right the time integrated anomaly $\beta^{(3)}$ given in (4.3).
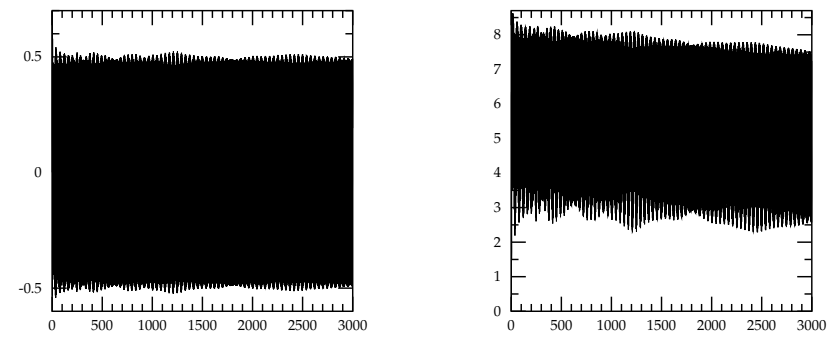

Figure 19. Anomalies for a breather-like configuration of the third class of models, defined in section 2.3 , for $\varepsilon=0.1$ and $\gamma=0.000$. On the left we show the anomaly $\alpha^{(3)}$ given in (4.2), and on the right the time integrated anomaly $\beta^{(3)}$ given in (4.3).

Of course, as is well known the modified sine-Gordon models do not possess breather solutions except for the sine-Gordon case. Thus, for all values of $\varepsilon$ the breather-like structures we have looked at gradually decay. This decay is carried out by the emission of radiation during the repetitive scattering of soliton and antisoliton forming the breather (i.e. the breathing of the breather-like configuration). Clearly, the speed of this decay, depends on $\varepsilon$ and $\gamma$. It also depends on $\nu$ - the frequency of the breather-like structure. We have studied this dependence and have observed that for (for a given $\nu$ ) the energy of the configuration decreases with the increase of $\varepsilon$ (it decreases by a factor of 3 from $\varepsilon=0.1$ to $\varepsilon=0.5)$. At the same time, for a given $\varepsilon$ and $\nu$ it increases with the increase of $\gamma$ and, like for the pure sine-Gordon model, for a given $\varepsilon$ and $\gamma$ it decreases with the increase of $\nu$. 

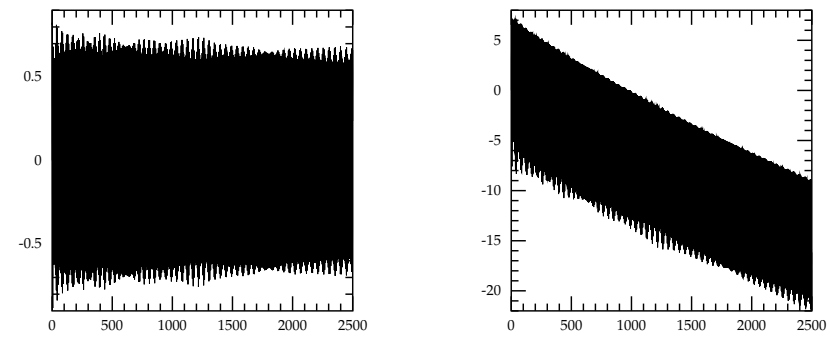

Figure 20. Anomalies for a breather-like configuration of the third class of models, defined in section 2.3 , for $\varepsilon=0.1$ and $\gamma=0.5$. On the left we show the anomaly $\alpha^{(3)}$ given in (4.2), and on the right the time integrated anomaly $\beta^{(3)}$ given in (4.3).
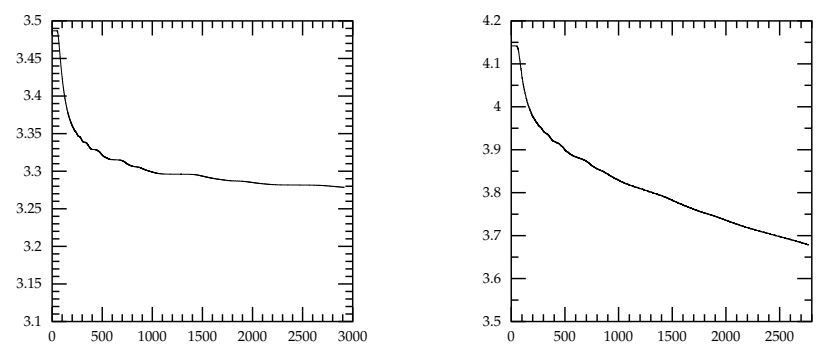

Figure 21. Total energy for breather-like configurations of the third class of models, defined in section 2.3. In the left plot we show the total energy as a function of time for $\varepsilon=0.1$ and $\gamma=0.0$. In the right plot we show the total energy as a function of time for $\varepsilon=0.1$ and $\gamma=0.5$.

These dependences have very important implications for the decay of the studied breather-like structures. Thus, in particular, if one keeps $\nu$ and $\varepsilon$ fixed the increase of $\gamma$ speeds up the decay of the structure, which for low values of $\gamma$ takes place gradually, while above a certain value of $\gamma$ happens quite suddenly. The value at which this happens depends on $\varepsilon$ and decreases with the increase of $\gamma$.

In figure 21 we present some preliminary plots of the decrease of the energy of two such breather-like configurations (both for $\nu=0.5$ and $\varepsilon=0.1$; one for $\gamma=0.0$ (the left hand-one) and one for $\gamma=0.5$.

They show the faster decrease of the configuration with $\gamma \neq 0$. 


\section{$5 \quad$ Further comments and some conclusions}

In this paper we have performed further studies of quasi-integrability - based on the observation $[1,2]$ that the anomaly, which distinguishes integrable models from non-integrable ones, also vanishes in some non-integrable models in which the field configurations possess the parity symmetries discussed in section 3 .

This observation was originally made in some very specific models and here we have tried to assess its general validity. So, we constructed three classes of models (one with symmetry, one without it and one (dependent on two parameters) which would allow us to interpolate between the two. Our results have confirmed the validity of our assumption (and so extended the class of models in which our observation holds) and have also allowed us to study the way the anomaly varies as we move away from the models with this extra symmetry.

Our work has also led us to look more clearly at breather-like configurations. Such configurations depend on many parameters and in this paper we have concentrated our attention at looking at the behaviour of such configurations when the symmetry is present and when it is absent. However, the symmetry is only one interesting topic to investigate for the breather-like configurations. One could look at the aspects of the decay of such configurations and the dependence of this behaviour of various parameters of these configurations.

The results reported in this paper support our claims about the importance of the extra symmetry and its influence on quasi-integrability. However, they have also shown that we have to understand our results better. Thus in the case of two solitons we would like to understand whether the change of $Q^{(3)}$ is mainly due to the change of velocity of moving solitons or to their perturbations. To be able to determine this we have to obtain results which are more accurate than those presented here. The same applies in the case of quasi-breathers. How do they lose their energy? To what extend this is dependent on all possible symmetries of our systems of solitons and how much their perturbations determine their evolutions. These are some of the topics we are looking at right now and we hope to be able to say something more definitive on these problems in not too distant future.

\section{Acknowledgments}

LAF is grateful for the hospitality at the Department of Mathematical Sciences of Durham University where part of this work was carried out. LAF and WJZ would like to thank the Royal Society for awarding them a grant which made their collaboration possible. LAF is partially supported by $\mathrm{CNPq}$ (Brazil). This paper was finished when the authors were "researching in pairs" at the Matematisches Forschunginstitute in Oberwolfach. They would like to thank the Oberwolfach Institute for its hospitality.

\section{A The algebra}

We consider the $\operatorname{sl}(2)$ algebra

$$
\left[T_{3}, T_{ \pm}\right]= \pm T_{ \pm}, \quad\left[T_{+}, T_{-}\right]=2 T_{3} .
$$


We take the following basis for the corresponding loop algebra

$$
b_{2 m+1}=\lambda^{m}\left(T_{+}+\lambda T_{-}\right), \quad F_{2 m+1}=\lambda^{m}\left(T_{+}-\lambda T_{-}\right), \quad F_{2 m}=2 \lambda^{m} T_{3} .
$$

The algebra is

$$
\begin{aligned}
{\left[b_{2 m+1}, b_{2 n+1}\right] } & =0, \\
{\left[F_{2 m+1}, F_{2 n+1}\right] } & =0, \\
{\left[F_{2 m}, F_{2 n}\right] } & =0, \\
{\left[b_{2 m+1}, F_{2 n+1}\right] } & =-2 F_{2(m+n+1)}, \\
{\left[b_{2 m+1}, F_{2 n}\right] } & =-2 F_{2(m+n)+1}, \\
{\left[F_{2 m+1}, F_{2 n}\right] } & =-2 b_{2(m+n)+1} .
\end{aligned}
$$

We have a grading operator

$$
d=T_{3}+2 \lambda \frac{d}{d \lambda}
$$

such that

$$
\left[d, b_{2 m+1}\right]=(2 m+1) b_{2 m+1}, \quad\left[d, F_{m}\right]=m F_{m} .
$$

Open Access. This article is distributed under the terms of the Creative Commons Attribution License (CC-BY 4.0), which permits any use, distribution and reproduction in any medium, provided the original author(s) and source are credited.

\section{References}

[1] L. Ferreira and W.J. Zakrzewski, The concept of quasi-integrability: a concrete example, JHEP 05 (2011) 130 [arXiv:1011.2176] [INSPIRE].

[2] L. Ferreira, G. Luchini and W.J. Zakrzewski, The concept of quasi-integrability for modified non-linear Schrödinger models, JHEP 09 (2012) 103 [arXiv:1206.5808] [INSPIRE].

[3] J. Hietarinta, Hirota's bilinear method and partial integrability, in Partially Integrable Evolution Equations in Physics, R. Conte and N. Boccara eds., NATO ASI Series C 310, Les Houches France March 21-30 1989 [INSPIRE].

[4] T. Kuusela and J. Hietarinta, Elastic scattering of solitary waves in the strongly dissipative Toda lattice, Phys. Rev. Lett. 62 (1989) 700.

[5] D. Bazeia, L. Losano, J. Malbouisson and R. Menezes, Classical behavior of deformed sine-Gordon models, Physica D 237 (2008) 937 [arXiv:0708.1740] [INSPIRE].

[6] C. Adam, L. Ferreira, E. da Hora, A. Wereszczynski and W. Zakrzewski, Some aspects of self-duality and generalised BPS theories, JHEP 08 (2013) 062 [arXiv:1305.7239] [INSPIRE].

[7] L. Ferreira and W.J. Zakrzewski, A simple formula for the conserved charges of soliton theories, JHEP 09 (2007) 015 [arXiv:0707.1603] [INSPIRE]. 